\title{
The Strong Confinement Regime in HgTe Two-Dimensional Nanoplatelets
}

Nicolas Moghaddam ${ }^{1}$, Charlie Gréboval ${ }^{2}$, Junling $\mathrm{Qu}^{2}$, Audrey $\mathrm{Chu}^{2}$, Prachi Rastogi², Clément Livache $^{2}$, Adrien Khalili', Xiang Zhen Xu ${ }^{1}$, Benoit Baptiste ${ }^{3}$, Stefan Klotz ${ }^{3}$, Guy Fishman ${ }^{4}$, Francesco Capitani $^{5}$, Sandrine Ithurria ${ }^{1}$, Sébastien Sauvage ${ }^{6^{*}}$, Emmanuel Lhuillier $^{2^{*}}$

'Laboratoire de Physique et d'Etude des Matériaux, ESPCI-ParisTech, PSL Research University, Sorbonne Université UPMC Univ Paris 06, CNRS, 10 rue Vauquelin 75005 Paris, France.

${ }^{2}$ Sorbonne Université, CNRS, Institut des NanoSciences de Paris, INSP, F-75005 Paris, France.

${ }^{3}$ Sorbonne Université, CNRS, Institut de Minéralogie, de Physique des Matériaux et de Cosmochimie, IMPMC, F-75005 Paris, France.

${ }^{4}$ Kadope, Centre de Nanosciences et de Nanotechnologies, 10 boulevard Thomas Gobert, 91120 , Palaiseau, France.

${ }^{5}$ Synchrotron-SOLEIL, Saint-Aubin, BP48, F91192 Gif sur Yvette Cedex, France.

${ }^{6}$ Université Paris-Saclay, CNRS, Centre de Nanosciences et de Nanotechnologies, 91120 , Palaiseau, France.

\begin{abstract}
The confinement in colloidal HgTe nanocrystals enables this material to be promising for colloidal optoelectronics over a wide range of energies, from the $\mathrm{THz}$ spectral range up to the visible region. Herein, by using a combination of high energy absorption $\mathrm{HgTe}$ nanoplatelets and low energy absorption $\mathrm{HgTe}$ nanocrystals, we probe optical transmission of $\mathrm{HgTe}$ nanoparticles over the $0.26-1.8 \mathrm{eV}$ range, from $0 \mathrm{~K}$ to $300 \mathrm{~K}$ temperatures and under simultaneous pressure, up to $4 \mathrm{GPa}$. While the pressure dependence of nanoplatelets follows the one observed for bulk and nanocrystals, the temperature dependence dramatically differs for nanoplatelets. The modeling of the electronic energy dispersion using up to 14-band k.p formalism suggests that the second conduction band and higher bands of HgTe play a vital role to describe and explain the HgTe nanoparticle spectroscopies.
\end{abstract}

*To whom correspondence should be sent: el@insp.upmc.fr and sebastien.sauvage@c2n.upsaclay.fr 


\section{INTRODUCTION}

In bulk semiconductors, spectral tunability relies on material alloying with a second compound. This alloying condition can become a challenge when lattice matched thin films need to be grown on a given substrate. In nanocrystals (NCs), quantum confinement offers an alternative method to achieve spectral control. This approach is nowadays used to generate green and red light for displays ${ }^{1}$ using $\mathrm{CdSe}$ or InP as active materials. In such application the confinement-induced renormalization of the band gap corresponds to a few tens of percent. Over the past decade, infrared optoelectronics ${ }^{2}$ have also generated interest with applications such as solar cells ${ }^{3,4}$ and low-cost infrared (IR) sensors ${ }^{5,6,7,8,9}$ including focal plane arrays. ${ }^{10,11,12}$ For these IR applications, narrow band gap materials such as lead and mercury chalcogenides are used, and the relative change of the band gap resulting from confinement can be even more important. Thus, the lowest energy optical feature of $\mathrm{HgTe}$ can be tuned from the $\mathrm{THz}^{13,14}$ to the visible range (i.e. over two orders of magnitude of energies) only using confinement, due to the lack of bulk band gap. To obtain such tunability the particle size needs to be tuned from a few monolayers ( $\approx 1 \mathrm{~nm}$ thickness) in HgTe two-dimensional (2D) nanoplatelets ${ }^{15,16}$ (NPLs) to $\approx 1 \mu \mathrm{m}$ diameter in bulk-like nanocrystals. ${ }^{14}$ The resulting change of confinement corresponds to very different ranges of wavevectors in the energy dispersion of the bulk material and raises the need for an accurate modelling of the energy dispersion all over the Brillouin zone.

The electronic structure of the bulk $\mathrm{Hg}(\mathrm{Cd}) \mathrm{Te}$ attracted significant interests in the 80's-90's owing to the use of this material for IR sensing. ${ }^{17,18}$ Bulk $\mathrm{HgTe}$ is a vanishing band gap semiconductor with an inverted band ordering, see Figure 1. ${ }^{19}$ Recently, the material regained some interest as the first example of a topological insulator. ${ }^{20}$ In the latter case, a barely confined quantum well of $\mathrm{HgTe}$ is sandwiched into a CdTe matrix and presents edge channels for transport, as the bands recover their normal ordering at the interface. ${ }^{21,22}$ For zero-dimensional (OD) NCs, the electronic spectrum has been deeply investigated using infrared spectroscopy to measure the absorption cross-section ${ }^{23}$, or to reveal the exciton fine structure. ${ }^{24,25}$ Complementary, the electronic spectrum of weakly confined HgTe NCs has been modelled using the two band k.p formalism ${ }^{28}$ and the tight binding method. ${ }^{26,27}$ However very little work have been devoted to the most strongly confined form of HgTe such as the one obtained in HgTe 2D NPLs where the confinement energy can be as large as $1.5 \mathrm{eV}$.

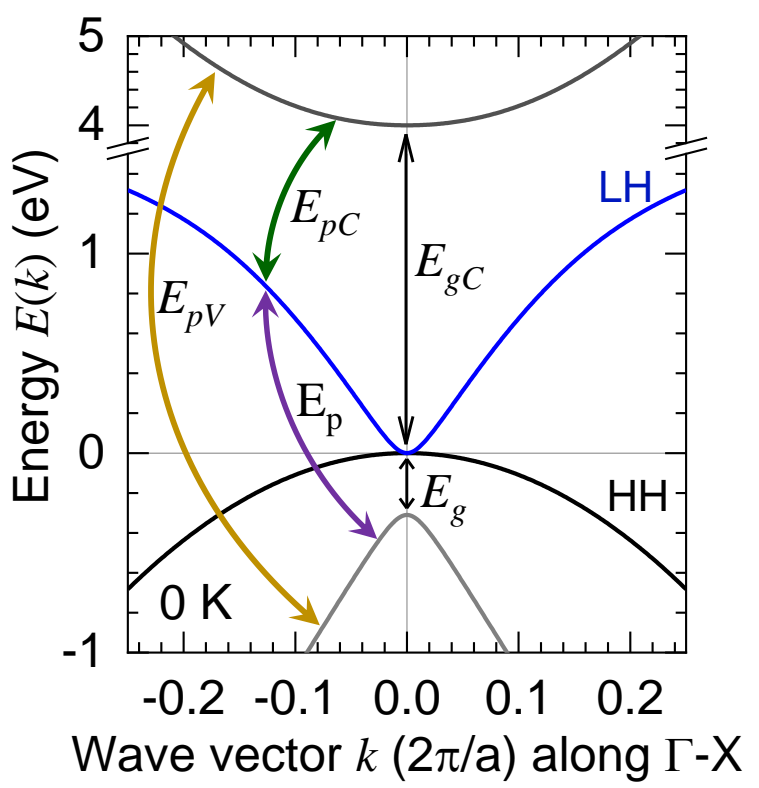

Figure 1 Dispersion relation $\boldsymbol{E}(\boldsymbol{k})$ of the $\mathrm{LH}$ conduction (blue) and $\mathrm{HH}$ valence (black) bands of bulk $\mathrm{HgTe}$ from the $(3+1)$ band $\boldsymbol{k} \cdot \boldsymbol{p}$ model

This lack appears to be detrimental since device progresses appear to be strongly correlated to a deeper understanding of the material and its electronic structure. For the sake of illustration, systematic measurements of the band alignment ${ }^{28,10,29}$ in $\mathrm{HgTe}$ NCs have enabled the design of unipolar barriers ${ }^{29}$ to 
reduce the device dark current. Alternatively, the development of $\mathrm{HgTe}$ based colloidal heterostructures appears promising to expand the reachable range of wavelength of the $2 \mathrm{D} \mathrm{NPLs,}{ }^{30,31}$ but also raises questions relative to the understanding of the optical spectrum to disentangle charge delocalization effect from pressure effect resulting from non-lattice matched shells. ${ }^{32}$

In this paper, we explore in a systematic way the effect of confinement, temperature and pressure on the energy dispersion of $\mathrm{HgTe}$. In practice, we grow a series of $\mathrm{HgTe} \mathrm{NCs}$ with various confinement energies from 0.26 to $1.8 \mathrm{eV}$. The temperature is tuned from $0 \mathrm{~K}$ to $300 \mathrm{~K}$ which corresponds to cryogenic operating condition for IR sensors and finally the pressure is tuned up to $4 \mathrm{GPa}$, corresponding to the full range of existence of the zinc blende low pressure phase of HgTe nanoparticles. While the pressure dependence of the spectroscopic properties appears to be weakly affected by the confinement, we show that the temperature dependence of the 2D NPLs is opposite to the one observed for bulk and weakly confined NCs. Our multiband k.p modelling points to the central role of the second conduction band in the origin of this observation. Finally, thanks to a 14 bands k.p model we are able to propose a set of parameters which correctly account for temperature and pressure dependence and describe the dispersion relation of $\mathrm{HgTe}$ over an extended range of the Brillouin zone.

\section{METHODS}

Chemicals: Mercury chloride $\left(\mathrm{HgCl}_{2}\right.$, Strem Chemicals, 99\%), $\mathrm{Hg}(\text { Acetate })_{2}\left(\mathrm{Hg}(\mathrm{Ac})_{2}\right.$ Aldrich, > $\left.99.0 \%\right)$, Mercury compounds are highly toxic. Handle them with special care. Tellurium powder (Te, SigmaAldrich, 99.99\%), cadmium oxide (CdO) (Aldrich 99.99\%), trioctylphosphine (TOP) (Alfa, $90 \%$ ), oleic acid (OA, Sigma, 90\%), propionic acid (Aldrich, $99 \%$ ), oleylamine (OLA, Acros, 80-90\%), dodecanethiol (DDT, Sigma-Aldrich, 98\%), Trioctylamine (TOA, Aldrich), Octadecene (ODE) (Aldrich, $90 \%$ ), chloroform (Carlo Erba), methanol (VWR, 98.5\%) acetone (VWR rectapur), ethanol (absolute VWR), toluene (VWR, 99.8 \%). All chemicals are used without further purification.

Cadmium propanoate (Cd(Prop) $\left.)_{2}\right): 1.036 \mathrm{~g}$ of $\mathrm{CdO}$ is mixed in $10 \mathrm{~mL}$ of propionic acid under Ar for 1 hour. Then the flask is open to air and the temperature risen to $140{ }^{\circ} \mathrm{C}$ up to the point the volume gets divided by a factor of two. The whitish solution is precipitated by addition of acetone. After centrifugation, the solid is dried under vacuum for 24 hours.

$1 \mathrm{M}$ TOP:Te precursor: $2.54 \mathrm{~g}$ of Te powder is mixed in $20 \mathrm{~mL}$ of TOP in a three neck flask. The flask is kept under vacuum at room temperature for $5 \mathrm{~min}$ and then the temperature is raised to $100^{\circ} \mathrm{C}$. Furthermore, degassing of flask is conducted for the next $20 \mathrm{~min}$. The atmosphere is switched to Ar and the temperature is raised to $275^{\circ} \mathrm{C}$. The solution is stirred until a clear orange coloration is obtained. The flask is cooled down to room temperature and the color switches to yellow. Finally, this solution is transferred to an Ar filled glove box for storage.

Synthesis of 3 MLs CdTe NPLs: The procedure follows the procedure proposed by Pedetti et al. ${ }^{33}$ In a three-neck flask, Cd(prop) 2 (390 mg, $1.5 \mathrm{mmol})$, oleic acid $(240 \mu \mathrm{L}, 0,75 \mathrm{mmol})$, and $30 \mathrm{~mL}$ of octadecene are degassed under vacuum at $90^{\circ} \mathrm{C}$ for 1 hour. Under argon flow, TOP:Te at $1 \mathrm{M}(300 \mu \mathrm{L})$ diluted in $1.5 \mathrm{~mL}$ of octadecene is swiftly injected at $210^{\circ} \mathrm{C}$. After 30 minutes of reaction, the mixture is cooled down and 1.7 $\mathrm{mL}$ of oleic acid is introduced. The resulting nanoplatelets are precipitated twice with an excess of ethanol and then are redispersed in $30 \mathrm{~mL}$ of hexane.

Cation exchange for synthesis of 3 MLs HgTe NPLs with TOA: In a glass tube, $2 \mathrm{~mL}$ of hexane and 200 $\mu \mathrm{L}$ of $\mathrm{HgTe}$ NPLs are introduced. $60 \mu \mathrm{L}$ of a fresh $0.1 \mathrm{M} \mathrm{HgAc}_{2}$ in trioctylamine solution is added at room temperature (The $\mathrm{Cd}: \mathrm{Hg}$ ratio is $1: 2$ ). The cation exchange is followed by absorption spectroscopy. Once the first excitonic peak has reached $824 \mathrm{~nm}, 80 \mu \mathrm{L}$ of oleic acid are added to stabilize the HgTe NPLs. The excess of precursor is removed by a two-step centrifugation $(6000 \mathrm{rpm}, 30 \mathrm{~s}$ and we keep the supernatant then $6000 \mathrm{rpm}, 5 \mathrm{~min})$. The resulting NPLs are resuspended in $1 \mathrm{~mL}$ of toluene. Procedures for other surface chemistry on the HgTe NPL surface are given in the SI 
HgTe NCs synthesis with band edge at $4000 \mathrm{~cm}^{-1}: 513 \mathrm{mg}$ of $\mathrm{HgCl}_{2}$ was added to $60 \mathrm{~mL}$ of oleylamine in a $100 \mathrm{~mL}$ round flask. The solution was placed under vacuum and heated to $110^{\circ} \mathrm{C}$ for $1 \mathrm{~h}$. Then, the temperature is decreased to $80^{\circ} \mathrm{C}$ and solution is placed to Ar atmosphere. $1.9 \mathrm{~mL}$ of TOP:Te (1 M) with 10 $\mathrm{mL}$ of oleylamine is added to the mercury solution. The solution color gradually turns to dark brown and the reaction is stopped at $3 \mathrm{~min}$. A solution made of $1 \mathrm{~mL}$ of dodecanethiol and $9 \mathrm{~mL}$ of toluene is quickly added to quench the reaction. The nanocrystals are then precipitated with ethanol. After centrifugation, the nanocrystals are redispersed in chloroform. The washing step is repeated one more time. The solution is filtered with a $0.2 \mu \mathrm{m}$ filter and redispersed in $6 \mathrm{~mL}$ of chloroform. Procedures for other NC sizes are given in the SI.

Electron microscopy: For transmission electron microscopy (TEM) pictures, a drop of the NC solution is drop-casted onto a copper grid covered with an amorphous carbon film. The grid is degassed overnight to reduce future contamination. A JEOL 2010F is used for acquisition of pictures and operated at $200 \mathrm{kV}$.

Infrared spectroscopy as a function of temperature: The solution of nanocrystal is drop-casted onto a double side polished $\mathrm{Si}$ wafer for the sample in the mid-infrared and onto a $\mathrm{CaF}_{2}$ substrate for the sample in the near-infrared. The sample is mounted on the cold finger of a closed cycle cryostat. The latter is used in a transmission configuration with two ZnSe windows. The cryostat is then introduced into the Fourier Transform Infrared spectrometer (FTIR, Thermo Fischer iS 50). Two sources have been used: a white light in the near infrared and a globar in the mid infrared. Two beam splitters have been used: a $\mathrm{CaF}_{2}$ beam splitter in the near infrared and an extended $\mathrm{KBr}$ in the mid infrared. Two detectors have been used: an InGaAs sensor in the near infrared and a DTGS in the mid infrared. In all case, the background is made at room temperature using the same optical configuration but without sample onto the substrate. Spectra are acquired with a 4 $\mathrm{cm}^{-1}$ resolution and typically averaged 100 times.

X-ray diffraction under pressure: Experiments were carried out using a membrane diamond anvil cell equipped with Boehler-type anvils with 500 micrometer culets. A stainless-steel gasket of 200 micrometer thickness was pre-indented to $70 \mu \mathrm{m}$, provided with a $200 \mu \mathrm{m}$ hole, and loaded with the sample, a 4:1 methanol-ethanol pressure transmitting medium and a $10 \mu \mathrm{m}$ diameter ruby sphere which served as pressure marker. The "Mao hydrostatic" pressure scale was used to determine pressures. X-ray powder diffraction measurements were performed at the XRD platform of the IMPMC on a Rigaku MM007HF diffractometer equipped with a Mo rotating anode $\left(\lambda_{\kappa \alpha 1}=0.709319 \AA, \lambda_{\kappa \alpha 2}=0.713609 \AA\right)$, Varimax focusing optics, and a RAXIS4++ image plate detector. X-ray data were collected at $20^{\circ} \mathrm{C}$. A LaB 6 standard sample was measured in the same experimental conditions to calibrate the Fit2D program, the image processing software used to integrate the intensities around the Debye-Scherrer rings and to get the 1D patterns.

Infrared spectroscopy under pressure: Membrane diamond anvil cells (DACs), equipped with Ilas-type diamonds having a 400-500 $\mu \mathrm{m}$ culets, are used. Stainless steel gaskets with a thickness of $200 \mu \mathrm{m}$ are indented down to $50 \mu \mathrm{m}$. A $150 \mu \mathrm{m}$ hole is then drilled by electro-erosion. The gasket is placed on the top of one of the DAC diamonds, and the gasket hole filled with $\mathrm{NaCl}$. After introducing a ruby crystal, the DAC is closed under gentle pressure until the salt forms a clear window. A drop of a diluted suspension of NCs in toluene is then added and dried on top of the $\mathrm{NaCl}$ window to from a dry and uniform film of NCs. The DAC is then closed and introduced in the Cassegrain microscope. the pressure is generated by the means of a metallic membrane inflated with He gas. The in-situ pressure is monitored by the ruby luminescence technique. A $532 \mathrm{~nm}$ laser is shone through one of the Cassegrain objectives and focused on the ruby crystal embedded in the cell. Ruby photoluminescence light is collected and sent to an OceanOptics spectrometer, showing a characteristic doublet around $694 \mathrm{~nm}$ at ambient pressure. This photoluminescence is fitted and the pressure is computed using the main peak position. Once the pressure is stabilized, an infrared absorbance spectrum is acquired using a Thermo Fisher Nicolet iS50 FTIR with a globar source, a quartz beam splitter, and a silicon detector in transmission configuration. This operation is repeated for various pressure from $0 \mathrm{GPa}$ to $4 \mathrm{GPa}$. When the maximum pressure is reached, the membrane is deflated and several spectra are acquired to check the reversibility of the pressure effect. 
k.p modeling and simulations: $3 \times 3$ and $14 \times 14$ k.p $H(k, T, P)$ hamiltonian matrices are processed symbolically and diagonalized numerically for each couple of temperature and pressure $(T, P)$ using the parameters in Table 1. Bulk material energy dispersion diagrams $E(k)$ are calculated using the wave vector $k$ as a free-varying independence variable. On the contrary, spectroscopic energies of the form $E(T, P)$ are extracted by modifying $k$, with the following procedure. Since thermal dilation or pressure contraction occurs, the nanoparticles are implicitly labelled by their discretized wave vector $k_{0}=2 \pi / L_{0}$ in $(0 \mathrm{~K}, 0 \mathrm{GPa})$ conditions. Based on the starting energy $E_{\text {Start }}$ in the starting conditions $\left(T_{\text {Start }}, P_{\text {Start }}\right)$, the dispersion relation $E(k)$ is first inverted giving out a reference wave vector $k_{\text {start }}$. This $k_{\text {Start }}$ wavevector is then dilated or contracted back to $(0 \mathrm{~K}, 0 \mathrm{GPa})$ conditions following equation $\mathrm{S} 6 \mathrm{~b}$, thus giving the $k_{0}$ wavevector labelling the nanoparticle. The temperature $T$ and pressure $P$ are then freely scanned. For each $(T, P)$, the norm of the discretized wave vector $k_{0}$ is changed into $k\left(k_{0}, T, P\right)$ according to the dilation or contraction of the lattice parameter. The k.p matrix is finally numerically evaluated at this altered wave vector $k$ and the eigen energies are reported against $T$ and/or $P$.

\section{RESULTS AND DISCUSSION}

\section{From weak to strong confinement in HgTe Nanocrystals}

We grow a series of HgTe NCs with various sizes to widely tune the confinement energy. As a strongly confined form of $\mathrm{HgTe}, 2 \mathrm{D}$ NPLs are grown. ${ }^{15}$ The HgTe NPLs are obtained using a two-step approach where CdTe NPLs are first synthetized, ${ }^{34}$ see Figure S1. The NPLs are 3.5 monolayers thick (3 planes of Te surrounded by 4 planes of $\mathrm{Cd}$ ) corresponding to a thickness of $1.1 \mathrm{~nm} \cdot{ }^{34}$ Those NPLs are then exposed to a bulky $\mathrm{Hg}$ precursor ( $\mathrm{Hg}$ oleate), which transforms them into pure $\mathrm{HgTe}$ NPLs due to cation exchange. ${ }^{15}$ Given that $\mathrm{CdTe}(0.648 \mathrm{~nm})$ and $\mathrm{HgTe}\left(0.646 \mathrm{~nm}^{35}\right)$ have similar lattice parameter $(0.3 \%$ difference $)$ the final thickness of the HgTe NPL is $1.1 \mathrm{~nm}^{15}$ which is confirmed by the fact that the two types of material present similar X-ray diffractogram, see Figure S2.

The lateral extension of the particles is large and can reach several hundred of $\mathrm{nm}$ according to electronic microscopy, see Figure 2a and S3-S5. Due to the thickness, the material is strongly confined along <001> crystal direction and the band edge absorption appears around $900 \mathrm{~nm}\left(\approx 11000 \mathrm{~cm}^{-1}\right.$ or $\approx 1.4 \mathrm{eV}$ at room temperature), see Figure 2c. It has been demonstrated that the exact value of the band-edge energy can be further tuned with the capping ligands which also take part in the effective delocalization length. ${ }^{15}$ This enables a fine tuning of the confinement energy while keeping the inorganic core unchanged.

In addition, we grow a series of HgTe NCs with room temperature band edge between $9000 \mathrm{~cm}^{-1}(1.11 \mathrm{eV})$ and $2000 \mathrm{~cm}^{-1}$ (248 meV), see Figure $2 \mathrm{~b}-\mathrm{c}$ and S6. The syntheses follow the procedure of Keuleyan et al. ${ }^{36}$ (for large NCs) and Geiregat et al. ${ }^{37}$ (for the smallest NCs). The combination of the HgTe NPLs and NCs allows us to tune the confinement energies over more than a factor of 5 , from strongly to weakly confined materials as we switch from NPLs to NCs. 

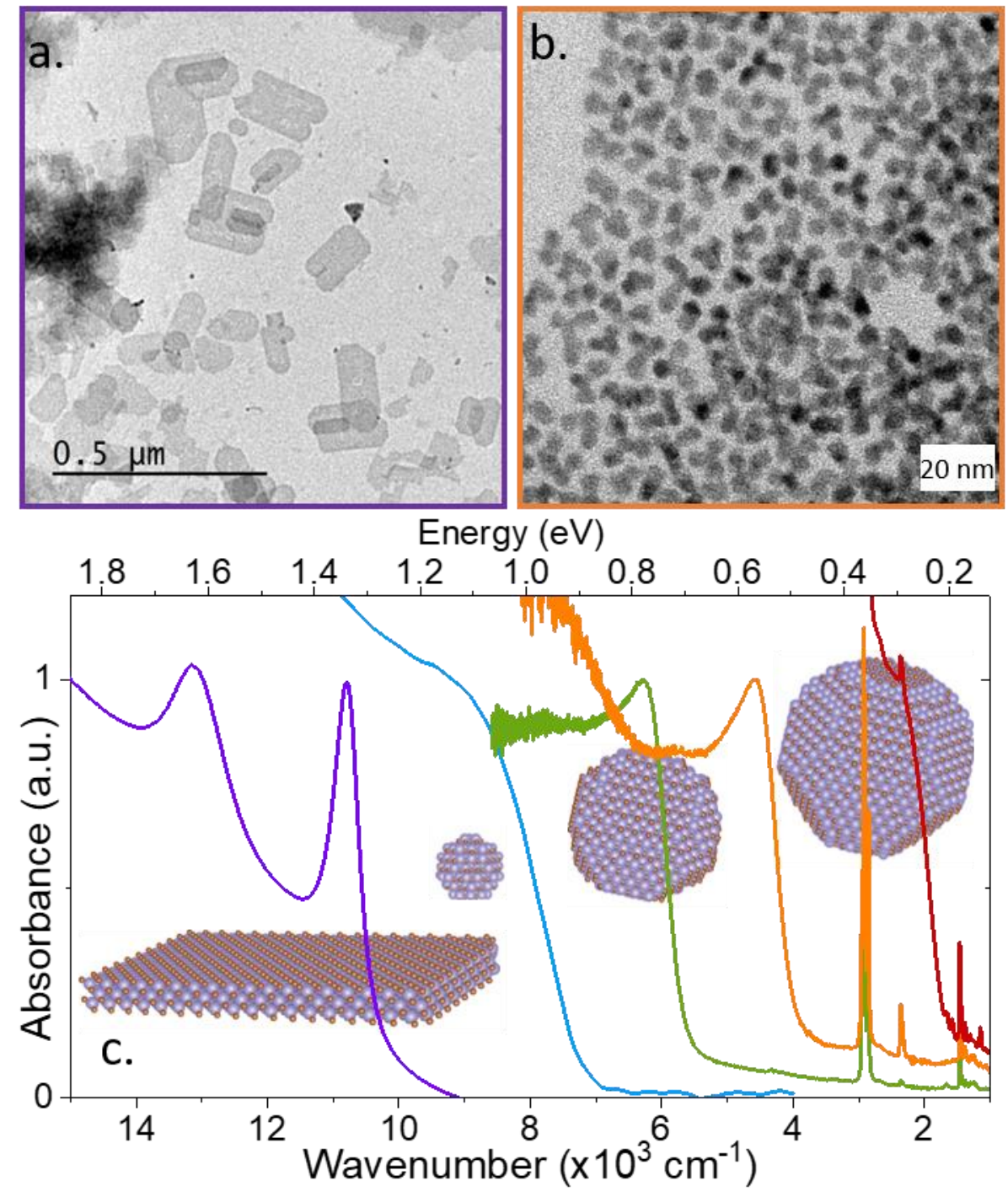

Figure 2 Confined NCs of HgTe. TEM images of a. HgTe NPLs and b. NCs. The associated absorption spectrum appears in purple and orange, respectively. c. Infrared absorption spectra for HgTe NPLs and NCs with various sizes.

\section{Effect of temperature of the infrared absorption}

We then probe the effect of temperature on the IR spectrum of the material, see the spectra in Figure 3a-c and S8-S9. The temperature dependence on the material is dramatically influenced by the material confinement, as reported in Figure 3a-c. For large (ie weakly confined) HgTe NCs (Figure 3c), the $E_{G}$ confinement energy redshifts as the temperature is lowered, corresponding to a positive value for $\mathrm{d} E_{G} / \mathrm{d} T$. This behavior is similar to the one observed in bulk $\mathrm{HgTe},{ }^{38,39}$ but is opposite to the trend observed for wider band gap II-VI semiconductors such as $\mathrm{CdTe}^{40}$ or $\mathrm{CdSe}^{41,42}$ where whatever the particle size only negative value have been observed for $\mathrm{d} E_{G} / \mathrm{d} T$. In NCs, such positive value for $\mathrm{d} E_{G} / \mathrm{d} T$ has been observed for lead halide perovskites ${ }^{43}$ or large PbS NCs. ${ }^{44}$ Nevertheless, as the confinement is increased, we observe a reduction of the value of $\mathrm{d} E_{G} / \mathrm{d} T$ (Figure $3 \mathrm{~b}$ ) and surprisingly even an inversion of its sign in the case of NPLs (Figure 3a). 

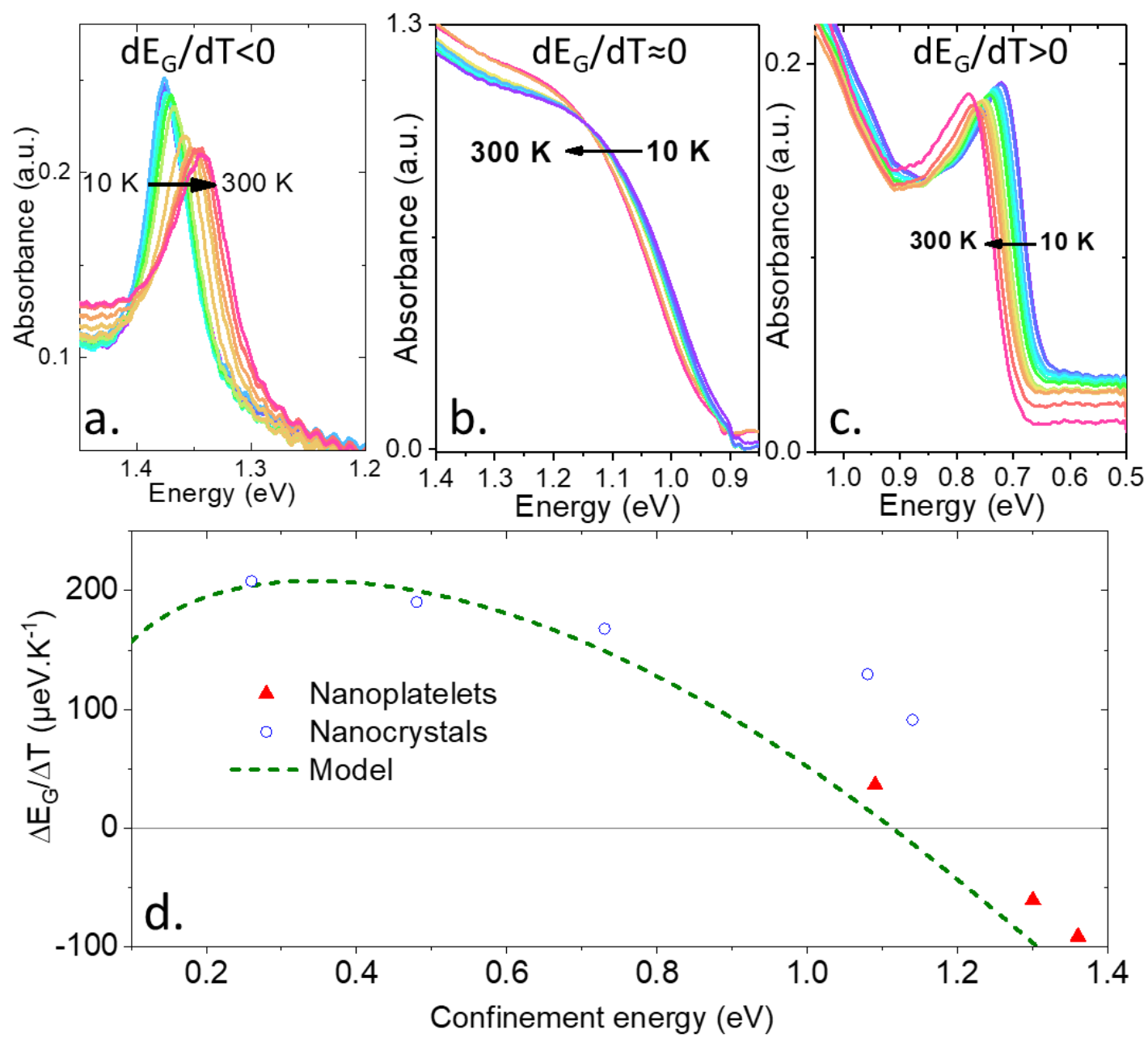

Figure 3 Effect of the temperature on the infrared spectra of HgTe NCs. a. Infrared absorption spectra for HgTe NPLs at various temperature between $10 \mathrm{~K}$ and $300 \mathrm{~K}$. The absorption blueshifts as temperature is lowered. b. Infrared absorption spectra for small $\mathrm{HgTe} \mathrm{NC}$ at various temperature between $10 \mathrm{~K}$ and $300 \mathrm{~K}$. The absorption is barely affected by temperature. c. Infrared absorption spectra for large HgTe NC at various temperature between $10 \mathrm{~K}$ and $300 \mathrm{~K}$. The absorption redshifts as temperature is lowered. $d$. Experimental temperature dependence of the band gap variation as a function of $\mathrm{HgTe} N C$ and NPL $E_{G}$ confinement energy (at $T=10 \mathrm{~K})$. The green dotted line is from the $(3+1) \mathrm{k} . \mathrm{p}$ model.

This change of sign for $\mathrm{d} E_{G} / \mathrm{d} T$ excludes that the observed shifts may result solely from a thermal dilation or contraction of the nanoparticles, the size of which will itself tune the confinement. In addition, this contribution of temperature has been found to be quantitatively weak ( $\mathrm{few} \mu \mathrm{eV} \cdot \mathrm{K}^{-123}$ ) due to the very small thermal dilation, see Figure S11b. Note that the dimensionality does not seems to be a driving parameter for the magnitude of $\mathrm{d} E_{G} / \mathrm{d} T$ since NCs and NPLs with the same $E_{G}$ confinement energy present similar values for $\mathrm{d} E_{G} / \mathrm{d} T$, see circles and triangles in Figure $3 \mathrm{~d}$. A similar trend for $\mathrm{d} E_{G} / \mathrm{d} T$ (reduction of the amplitude and change of sign) as a function of the inverse of the particle size was observed in the case of PbS NCs. By excluding other possibility, the band gap shift with temperature was finally attributed to a size dependent electron-phonon coupling. ${ }^{23,44}$

\section{Effect of pressure of the infrared absorption}

Similarly, we measured the pressure dependence of the band gap for the HgTe NPLs at various fixed temperatures. Note that for HgTe NCs the pressure dependence of the band gap was already reported in ref ${ }^{45}$ at room temperature. This is why the effect of pressure is discussed in the case of NPL here. In practice, the sample is loaded in a diamond anvil cell together with $\mathrm{NaCl}$ which is used as an infrared transparent pressure transmission medium. A ruby is also loaded in the cell and used to determine the applied pressure, 
see Figure 4a for a scheme of the experiment and the Supporting Information (SI) for details. All the reported observations appear to be fully reversible in the targeted range of pressure (i.e. zero pressure spectrum is recovered after pressure releasing). On the other hand, the process is strongly hysteretic: the full release of pressure is required to recover the initial spectrum.

To understand pressure induced change in the infrared spectrum, it is first necessary to determine if the changes might be the result of structural phase transition. Bulk $\mathrm{HgTe}$ crystallizes in the cubic zinc blende structure at ambient pressure and undergoes several phase changes as pressure is increased. For bulk material, the cinnabar phase appears around $1.4 \mathrm{GPa} .{ }^{46,47}$ Under NC form, the zinc-blende phase remains stable up to higher pressures. ${ }^{45}$ In the range between 3 and $5 \mathrm{GPa}$, we can notice the presence of both zinc blende and cinnabar phase (Figure S7). It is however unclear if the two phases exist within the same NPL or if the signal is associated with different particles. In the zinc-blende phase, we identify a decrease in the lattice parameter with initial pressure $(\mathrm{d} a / \mathrm{d} \approx \approx 7 \mathrm{pm} / \mathrm{GPa})$, which corresponds to a bulk modulus of $30 \mathrm{GPa}$ for the HgTe NPLs. This value is similar to the one measured for the $\mathrm{HgTe} \mathrm{NCs}^{47}$ (33 GPa) which was relatively close to the one of the bulk (34 GPa). ${ }^{48}$

The $E_{G}$ confinement energy appears to be dependent on the applied pressure, see Figure $4 \mathrm{~b}-\mathrm{c}, 6 \mathrm{c}$ and $\mathrm{S} 10$. The confinement energy gradually increases with pressure corresponding to a positive value for $\mathrm{d} E_{G} / \mathrm{d} P$. This is similar to what have been measured for HgTe NCs, ${ }^{45}$ and wider band gap II-VI semiconductor quantum dots such as $\mathrm{CdSe}^{49}$ and core shell made of $\mathrm{CdSe} / \mathrm{ZnS} .{ }^{50,51}$ Applying pressure in the range of existence of the zinc blende phase can leads to a shift of the confinement energy up to $300 \mathrm{meV}$, which is far more than the shift induced by cooling the sample from room temperature to cryogenic condition ( $\approx 35 \mathrm{meV})$. This order of magnitude is partially expected from the lattice parameter change under pressure around $6 \%$ in Figure S7c and estimated around only $0.08 \%$ over $300 \mathrm{~K}$ temperature range in Figure S11b. Above $4 \mathrm{GPa}$, no excitonic feature is identified. This observation is correlated to the disappearance of the zinc-blende phase under similar pressure, see Figure S7. 

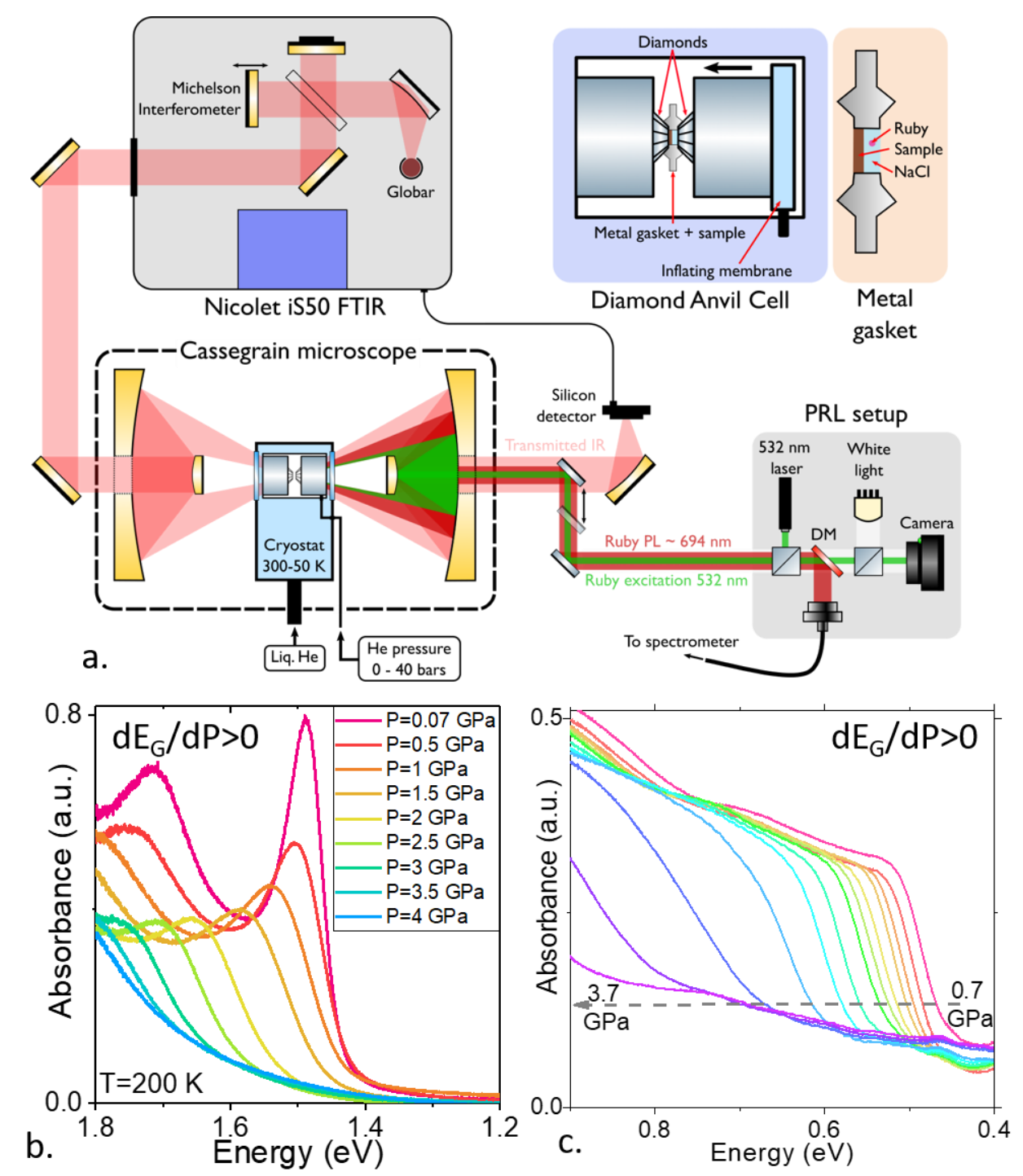

Figure 4 Effect of the pressure on the infrared spectra of HgTe NCs. a. Scheme of the experimental setup of the SMIS beamline of synchrotron SOLEIL to probe the infrared spectrum of a HgTe NPLs under pressure. b. Infrared spectrum of HgTe NPLs at $200 \mathrm{~K}$ and under various pressure in the range of 0 to $4 \mathrm{GPa}$. c. Infrared spectrum of $\mathrm{HgTe} \mathrm{NCs}$ at $300 \mathrm{~K}$ and under various pressure in the range of 0 to $4 \mathrm{GPa}$

\section{Multiband k.p modelling}

In order to address the striking temperature and pressure dependence of the spectra observed in Figure 3 and Figure 4, we model the nanoparticles energies using multiband k.p simulations. HgTe dispersion appears to be non-parabolic ${ }^{26,52}$ and the effective mass approximation ( 1 band model) is only valid over $1 \%$ of the Brillouin zone. For wavevector far away for the $\Gamma$ point, the dispersion becomes first linear and then further slightly bends down for wavevectors corresponding to the confinement of NPL (confinement energy above $1 \mathrm{eV}$ ). More bands need to be included in the k.p model to obtain an accurate description of the dispersion relation.

When a detailed description of the valence band is required, 8 band k.p model describing the $\Gamma_{6}, \Gamma_{7}$ and $\Gamma_{8}$ bands and their degeneracy is used. ${ }^{53,54}$ It has been estimated that the 8 band k.p model correctly described the Brillouin zone over $8 \%$ of it, corresponding to energies significantly smaller than the ones of the NPLs which are around 1.1-1.8 eV. ${ }^{53}$ While, the effective mass approximation only describes the very bottom parabolic part of the light hole like $\left(\Gamma_{8}\right)$ band, the inclusion of additional conduction band is critical to capture the linear dispersion occurring at larger wavevector and higher energies. 
In HgTe contrary to PbS, the hole and electron effective masses strongly differ, the valence band mass being more than 10 times the conduction band mass. So the confinement is mostly the result of the conduction band and the latter is the one requiring the most accuracy. In the SI (Figure S14-S17), we provide a 14-band k.p model, which includes the minimum amount of bands to properly take into account additional conduction bands. ${ }^{55}$

This 14-band model aims at supporting a simpler $(3+1)$ effective bands model later described in the main text. This simple model provides the driving concepts leading to the realistic calculated 14-band structure. The 14-band model includes notably the spin-orbit coupling, disregarded in the simplified model, and all relevant interactions. Note that coulombic renormalization of the band gap has been neglected since these effects have found to be small in the case of $\mathrm{HgTe} \mathrm{NCs.}{ }^{26}$ Even though the conclusions are qualitatively the same using each version of the model, the complete version may somehow blurry the message with a large amount of involved parameters. This is why, in the following, we describe a simplified approach. We use a $(3+1)$ bands one-dimensional k.p-like model, referred to as the simple model and described in the SI. Its illustrative role is to be tractable and to account for all the experimental features with a minimal set of necessary ingredients, defined in Figure 1. The associated parameters are given in Table 1.

The $(3+1)$ band model accounts for the 2 bands with $\Gamma_{8}$ symmetry at $\vec{k}=0$ (called HH and LH in Figure 1) which are the bands around the Fermi level and involved in the absorption. We also include the $\Gamma_{6}$ valence band and a second conduction band. The introduction of this effective high energy band in the conduction band is central to accurately describe the bending of the LH band occurring at large wave vector.

All the experimental data are then accounted from the variation with temperature and pressure of only 2 bulk band gap energies: $E_{g}(T, P)$ and $E_{g c}(T, P)$. As these gaps will be affected by pressure and temperature, the bands curvature will be modified (through the $E_{p}$ and $E_{p c}$ parameters coupling the bands), unveiling effect of pressure and temperature in presence of confinement (i.e. away from the $\Gamma$ point). The strong confinement in the HgTe nanoparticles is modeled by the discretization of the $\vec{k}$ wavevector in the reciprocal space, typically at $k= \pm \frac{\pi}{L}$ along [001] where $L$ is size of the nanoparticle, accounting for the contraction and thermal dilation of the nanoparticle with pressure and temperature respectively.

Table 1 Values of the simple $(3+1)$ and the 14-band model parameters used to describe the confinement, temperature and pressure dependences. n.a. means non applicable.

\begin{tabular}{|c|c|c|c|}
\hline Parameter & $\begin{array}{ll}\begin{array}{l}\text { Simple } \\
\text { band } \\
\text { value }\end{array} & \text { model } \\
\end{array}$ & $\begin{array}{l}\text { 14-band } \\
\text { model value }\end{array}$ & Reference \\
\hline$E_{g}^{0}$ & \multirow{2}{*}{$0.31 \mathrm{eV}$} & $0.375 \mathrm{eV}$ & \multirow{2}{*}{$0.303 \mathrm{eV}$ in Ref. 56} \\
\hline Simulated $E_{g}(k=0)$ & & $0.305 \mathrm{eV}$ & \\
\hline$E_{g C}^{0}$ & \multirow{2}{*}{$4 \mathrm{eV}$} & $2.5 \mathrm{eV}$ & \multirow{2}{*}{$\begin{array}{lll}4.11 & \text { eV } & \text { (QSGW) } \\
3.07 \text { eV (LDA) in Ref. 52 }\end{array}$} \\
\hline Simulated $E_{g C}(k=0)$ & & $2.78 \mathrm{eV}$ & \\
\hline$E_{p}$ & $25 \mathrm{eV}$ & $30 \mathrm{eV}$ & Fitted, $24.8 \mathrm{eV}$ in Ref. 56 \\
\hline$E_{p C}$ & $15 \mathrm{eV}$ & $20 \mathrm{eV}$ & Fitted \\
\hline$E_{p V}$ & $2.5 \mathrm{eV}$ & $10 \mathrm{eV}$ & Fitted \\
\hline$\partial E_{g} / \partial T$ & $\begin{array}{l}\text { from } \\
E_{g}(T, P) \text {, typically } \\
\approx-0.61 \text { meV.K } \\
1\end{array}$ & $\begin{array}{l}\text { from } E_{g}(T, P) \text { plus } \\
\text { a constant } 0.1 \\
\text { meV. } \mathrm{K}^{-1} \text {, typically } \\
\approx-0.51 \quad \text { meV. } \mathrm{K}^{-1}\end{array}$ & See $E_{g}(T, P)$ formula in SI \\
\hline$\partial E_{g C} / \partial T$ & $-2.4 \mathrm{meV} . \mathrm{K}^{-1}$ & $-3 \mathrm{meV} . \mathrm{K}^{-1}$ & Fitted \\
\hline$\partial E_{g} / \partial P$ & $-85 \mathrm{meV} / \mathrm{GPa}$ & $-95 \mathrm{meV} / \mathrm{GPa}$ & Fitted \\
\hline$\partial E_{g C} / \partial P$ & $+220 \mathrm{meV} / \mathrm{GPa}$ & $+600 \mathrm{meV} / \mathrm{GPa}$ & Fitted \\
\hline
\end{tabular}




\begin{tabular}{|c|c|c|c|}
\hline $\begin{array}{l}a \text { bulk } \mathrm{HgTe} \text { lattice } \\
\text { parameter }\end{array}$ & \multicolumn{2}{|c|}{$0.6465 \mathrm{~nm}$} & Ref 35 at $300 \mathrm{~K}$ \\
\hline $\begin{array}{l}a_{0}^{N P} \text { HgTe nanoparticule } \\
\text { lattice parameter }\end{array}$ & \multicolumn{2}{|c|}{$0.6500 \mathrm{~nm}$} & $\begin{array}{l}\text { From measurement Fig. S6c on } \\
\text { NPLs at } 300 \mathrm{~K}\end{array}$ \\
\hline $\begin{array}{l}B_{0} \quad \mathrm{HgTe} \text { nanoparticule } \\
\text { elasticity modulus }\end{array}$ & \multicolumn{2}{|c|}{$30 \mathrm{GPa}$} & $\begin{array}{l}\text { From measurement Fig. S6c on } \\
\text { NPLs at } 300 \mathrm{~K}\end{array}$ \\
\hline$\partial B_{0} / \partial P$ & \multicolumn{2}{|c|}{-1.5} & $\begin{array}{l}\text { From measurement Fig. S6c on } \\
\text { NPLs at } 300 \mathrm{~K}\end{array}$ \\
\hline$m_{L H}^{*}$ & $0.013 m_{0}$ & $0.013 m_{0}$ & Output \\
\hline $\mathrm{m}_{\mathrm{HH}}^{*}$ & $0.33 m_{0}$ & $0.69 m_{0}$ & Output \\
\hline Spin-orbit coupling $\Delta$ & n.a. & $0.5 \mathrm{eV}$ & \multirow{2}{*}{$\begin{array}{l}0.775 \text { eV (hQSGW) in Ref. } 52 \\
1.08 \text { eV in Ref. } 52\end{array}$} \\
\hline $\begin{array}{l}\text { Simulated spin-orbit } \\
\text { coupling at } k=0\end{array}$ & n.a. & $0.732 \mathrm{eV}$ & \\
\hline$\Delta_{C}$ & n.a. & $1 \mathrm{eV}$ & \multirow{2}{*}{$\begin{array}{l}1.06 \mathrm{eV} \text { (QSGW) } 1.17 \mathrm{eV} \\
\text { (LDA) in Ref. } 52\end{array}$} \\
\hline $\begin{array}{l}\text { Simulated spin-orbit } \\
\text { coupling at } k=0\end{array}$ & n.a. & $0.768 \mathrm{eV}$ & \\
\hline$\Delta^{\prime}$ & n.a. & $-1.5 \mathrm{eV}$ & Fitted \\
\hline
\end{tabular}

Figure 5a and Figure 6a show respectively the effects of temperature and pressure on the relation dispersion. When temperature or pressure increases, the band gaps $E_{g}$ and $E_{g c}$ are altered as depicted in Figure S11c. The effect on the LH band effective mass $m_{L H}^{*}$, defined at small $\mathrm{k}$, is given by:

$$
E_{L H}(k, T, P) \simeq\left(1+\frac{E_{p}}{E_{g}(T, P)}-\frac{E_{p C}}{E_{g C}(T, P)}\right) \frac{\hbar^{2} k^{2}}{2 m_{0}}=\frac{\hbar^{2} k^{2}}{2 m_{L H}^{*}}
$$

As seen in the equation (1), these variations lead to a competition between the two gaps $E_{g}(T, P)$ and $E_{g C}(T, P)$ in the alteration of $E_{L H}(k)$ relation dispersion.

At low energy (ie small $\mathrm{k}$, see the left arrow in the dispersion relation) the dispersion is ruled by the $m_{L H}^{*}$ effective mass reduction. The energy blue-shifts as shown in Figure 5a for temperature, and Figure 6a for pressure as the result of the drastic reduction of $E_{g}$ from $0.31 \mathrm{eV}$ at $0 \mathrm{~K}$ down to $0.12 \mathrm{eV}$ at room temperature. $E_{g}$ being one order of magnitude smaller than $E_{g C}$, and $\partial E_{g} / \partial T / E_{g}$ and $\partial E_{g C} / \partial T / E_{g C}$ partial derivatives being comparable, the effective mass is essentially governed by $E_{g}$ (i.e. the second conduction band plays no role in the vicinity of the $\Gamma$ point). The same rule of thumb holds for pressure.

However, at high energies (i.e. high $\mathrm{k}$, see the right arrow in the dispersion relation), the parabolic equation (1) ceases to be valid. When temperature increases, the second conduction band counteracts $m_{L H}^{*}$ reduction. As the result of the shrinking of $E_{g C}$, the second conduction band becomes closer to the LH band and repeals it downwards as seen in Figure 5a. In other words, the second conduction band is responsible for the sublinear relation dispersion observed at large $k$ and leads to an inversion of the confinement energy variation with a simulated red-shift of the $E_{G}$ confinement energy.

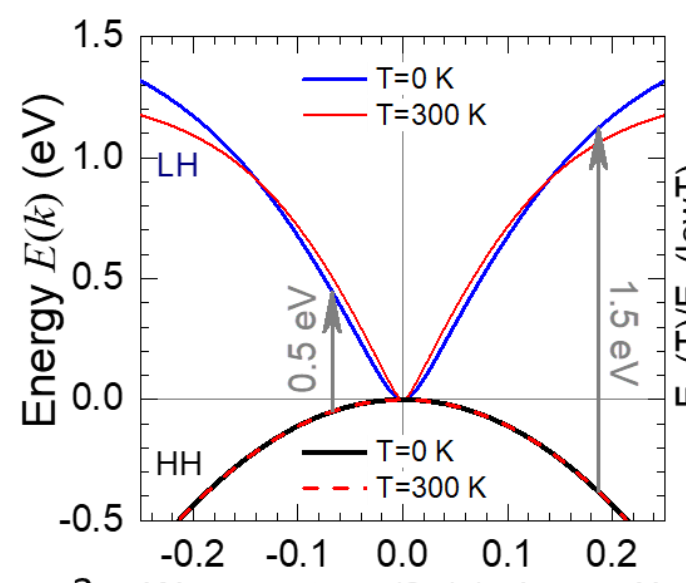

a. Wave vector $k(2 \pi / a)$ along $\Gamma-X$
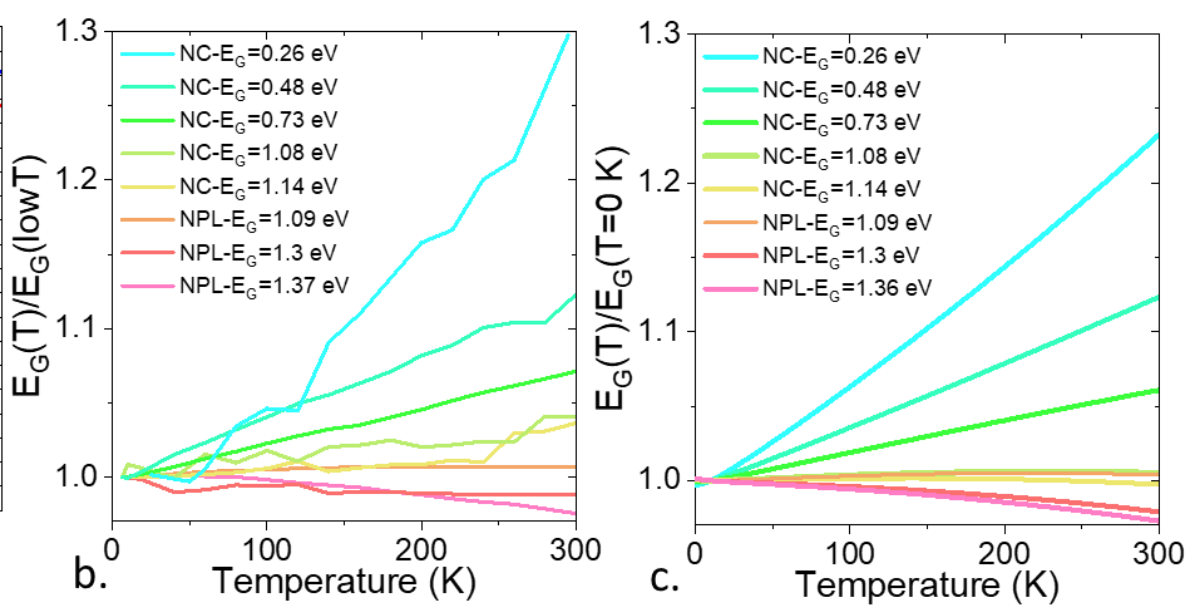
Figure 5 Modelling the temperature effect of the dispersion relation. a. Effect of temperature on $E(k)$ when the two bands gaps $E_{g}$ and $E_{g c}$ varies. The gray arrows corresponds to typical confinement energies for the NPL $(1.5 \mathrm{eV})$ and for NCs $(0.5 \mathrm{eV})$. b. Experimental normalized shift of the band gap as a function of temperature for different $10 \mathrm{~K}$ sizes of HgTe NCs and $3 \mathrm{ML} \mathrm{HgTe} \mathrm{NPLs}$ with different surface chemistry. $\mathrm{C}$. Simulated normalized shift of the confinement energy $E_{G}$ as a function of temperature for different sizes of $\mathrm{HgTe}$ NCs and $3 \mathrm{ML} \mathrm{HgTe}$ NPLs with different surface chemistry.

Using the $(3+1)$ band model and $-2.4 \mathrm{meV} . \mathrm{K}^{-1}$ value for $\frac{\partial E_{g C}}{\partial T}$, the experimental temperature dependence of the band gap (Figure 5b) is reproduced, as shown in Figure 5c. With low $T=10 \mathrm{~K}$, the blue shift $\left(E_{G}(T) / E_{G}(\right.$ low $\left.T)>1\right)$ at small energies is due to the shrinking of the $E_{g}$ band gap. However, at large energies, $E_{g C}$ also shrinks and the closer second conduction band pushes the first one downward, leading to a redshift $\left(E_{G}(T) / E_{G}(\right.$ low $\left.T)<1\right)$. This interpretation is further analyzed quantitatively by plotting the simulated slopes $\frac{\Delta E_{G}}{\Delta T}$ as a function of the confinement energy of the particles, see the dashed green line in Figure $3 \mathrm{~d}$. We see that the model appears in fair agreement with the observed slopes over the whole range of experimental energies supporting the interpretation of the shrinking of the two gaps energies.

Regarding the pressure dependence, the observed energy shifts with hydrostatic pressure present an average slope $\frac{\Delta E_{G}}{\Delta P} \simeq 100 \mathrm{meV} / \mathrm{GPa}$ in the case of NPL (Figure $4 \mathrm{~b}$, Figure $6 \mathrm{c}$, and $\mathrm{S10}$ ) which has to be compared with the value of $\approx 60 \mathrm{meV} / \mathrm{GPa}$ for the nanocrystals ${ }^{45}$ (Figure $4 \mathrm{c}$ and $\mathrm{S} 13$ ). Contrary to the case of temperature, the confinement only quantitatively affects the pressure dependence of the confinement energy. This result contrasts to CdSe where the size dependence of the $\frac{\Delta E_{G}}{\Delta P}$ parameter has been found to be larger with an increase of $\frac{\Delta E_{G}}{\Delta P}$ from 40 to $80 \mathrm{meV} / \mathrm{GPa}$ as the size changes by only a factor two. ${ }^{49}$

Under increasing pressure, the second conduction band goes away from the first one. Pressure simply pushes $E_{G}$ confinement energy even higher, see Figure $6 \mathrm{a}$. In this case, due to pressure, the second conduction band adds an additional contribution to the one of $E_{g}$ to the observed blue-shift of the confinement energy $E_{G}$. 


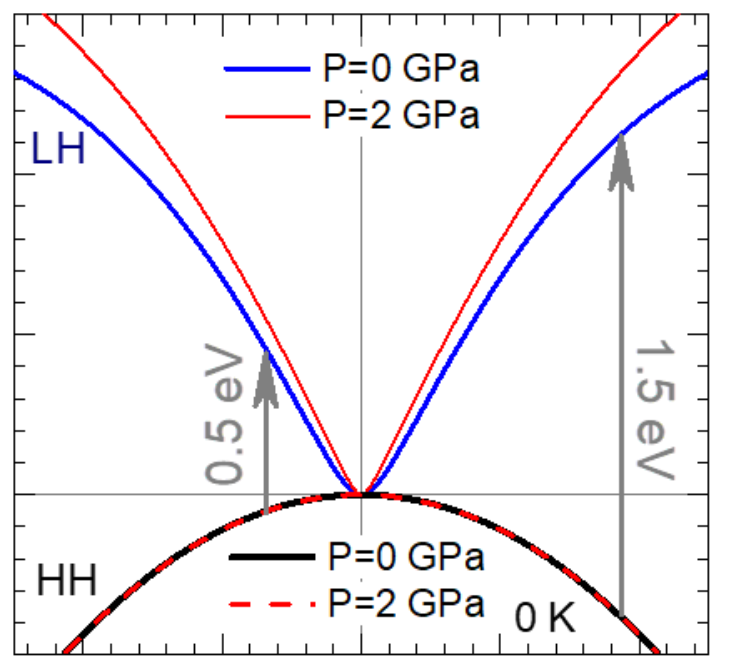

a. $-\begin{array}{ccccc}-0.2 & -0.1 & 0.0 & 0.1 & 0.2\end{array}$ Wave vector $k(2 \pi / a)$ along $\Gamma-X$

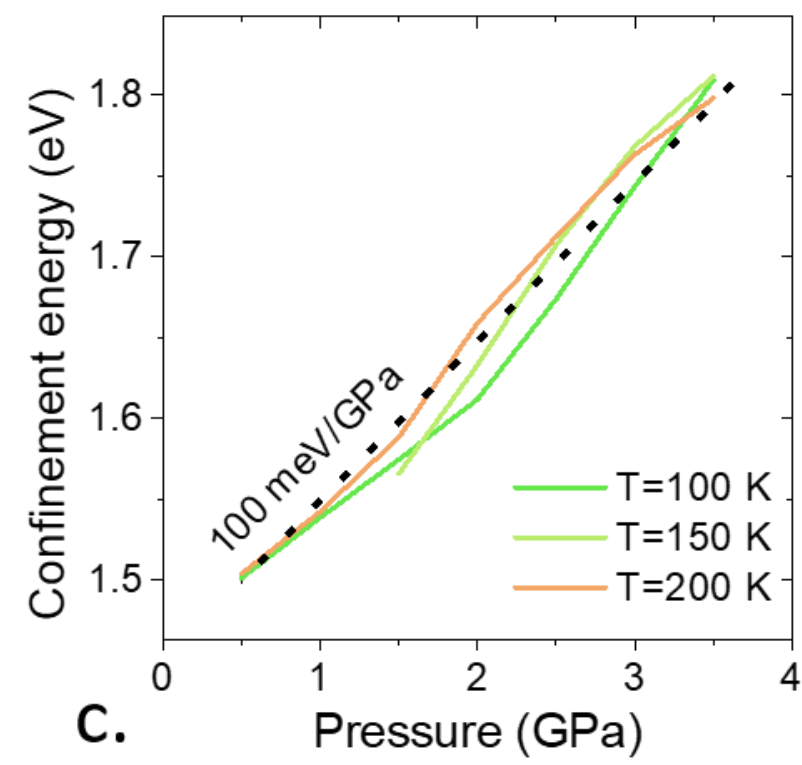

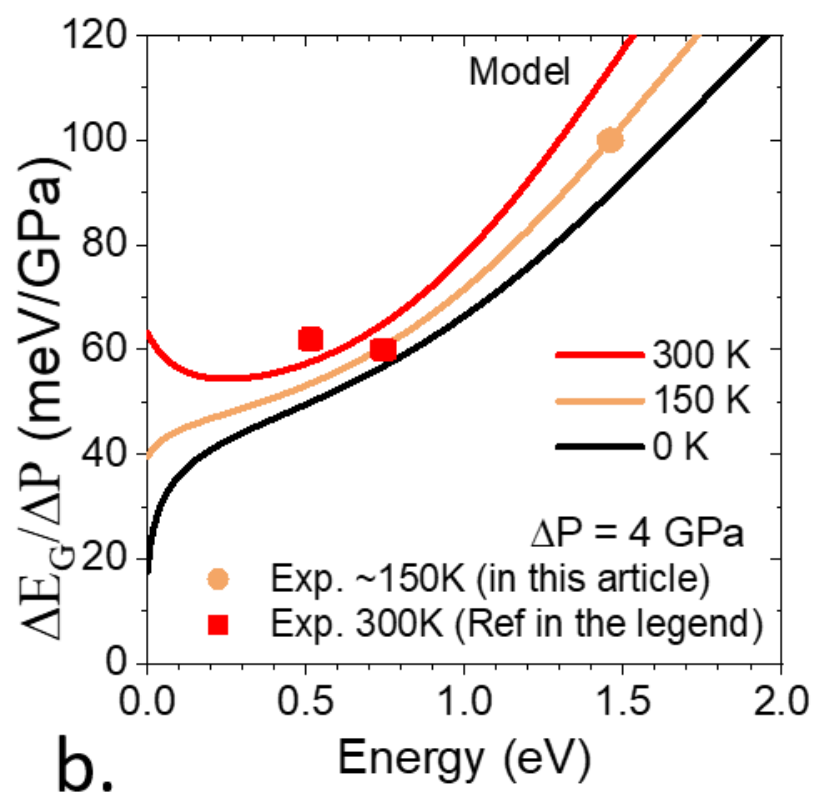

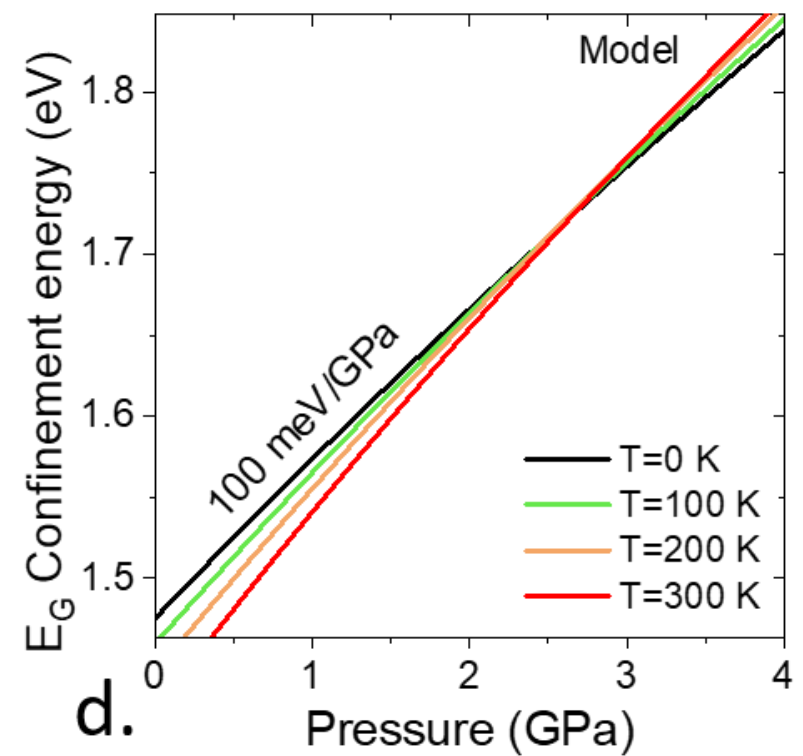

Figure 6 Modelling the pressure effect of the dispersion relation a. Effect of pressure on the conduction and valence bands. The gray arrows corresponds to typical confinement energies for the NPL (1.5 eV) and for NCs $(0.5 \mathrm{eV})$. b. Simulated confinement energy variation over $\triangle P=4 \mathrm{GPa}$ pressure difference for 3 temperatures. The light orange dot is the experimental measurement around $150 \mathrm{~K}$. The two red squares are the measurements from Ref ${ }^{45}$ at $300 \mathrm{~K}$. c. Experimental confinement energy $E_{G}$ shift as a function of an applied hydrostatic pressure for HgTe NPLs for three different temperatures. $d$. Simulated confinement energy $E_{G}$ shifts as a function of an applied pressure for HgTe NPLs from OK to 300K. The indicated slope corresponds to $0 \mathrm{~K}$

Using $-85 \mathrm{meV} / \mathrm{GPa}$ and $+220 \mathrm{meV} / \mathrm{GPa}$ respectively for $\partial E_{g} / \partial P$ and $\partial E_{g C} / \partial P$, the $(3+1)$ bands model can reproduce the pressure dependence of the confinement energy of the NPL, see Figure $6 c$ and d. Also note that using the temperature dependence determined previously, it is possible to reproduce the weak temperature dependence of the pressure potential $d E_{G} / d P$ of the confinement energy in the case of NPL, see the four simulated temperatures in Figure $6 \mathrm{~d}$, while capturing the moderate confinement dependence as depicted in Figure 6b and S13a.

In all, though both temperature and pressure alter the bulk band gaps $E_{g}$ and $E_{g C}$, only blue shifts are observed with increasing pressure, while increasing temperature also shows red-shift. We thus conclude that the unusual change of the optical band gap with temperature and its change of sign reflects the bulk material electronic structure and that phonon does not have to be invoked. ${ }^{44}$ 


\section{CONCLUSION}

In conclusion, we have shown that the strong confinement in NPL causes a non-linear dispersion of high energy state energies in the electronic structure of $\mathrm{HgTe}$ nanoparticles. Measurements of the temperature dependence of the optical band gap appears as a direct probe of the dispersion relation. We found that a single parameter set in the $(3+1)$ effective band model can account for all the observed features in energy, temperature, and pressure, providing that the relative proximity of the second conduction band of $\mathrm{HgTe}$ be included. We show that the second conduction band appears as a paramount ingredient for absorption energies above $\approx 0.5-1 \mathrm{eV}$ to describe realistically the energies and electronic states of strongly confined HgTe nanoparticles.

\section{ASSOCIATED CONTENT}

\section{Supporting information}

The Supporting Information is available free of charge at

Supporting Information include details about material synthesis, material characterization, X-ray diffraction measurement under pressure, raw spectrum about material temperature and pressure dependence and additional information regarding the (3+1)-band k.p-like and 14 band k.p models.

\section{ACKNOWLEDEGMENTS}

We thank Mayank Goyal for participation to synchrotron experiments. This work has been supported by the Region Ile-de-France in the framework of DIM Nano-K (grant dopQD). This work was supported by French state funds managed by the ANR within the Investissements d'Avenir programme by Labex Matisse (ANR11-IDEX-0004-02), and Labex NanoSaclay (ANR-10-LABX-0035), and also by the grant FRONTAL (ANR19-CE09-0017), IPER-Nano2 (ANR-18CE30-0023-01), Copin (ANR-19-CE24-0022), Graskop (ANR-19CE09-0026). JQ thanks Chinese Scholar council for PhD grant while AC thanks Agence Innovation Defense. EL thanks the support ERC starting grant blackQD (grant $n^{\circ} 756225$ ). SI thanks the support ERC starting grant Ne2Dem (grant $n^{\circ}$ 853049).

\section{REFERENCES}

(1) Bourzac, K. Quantum Dots Go on Display. Nature 2013, 493, 283.

(2) Green, M.; Mirzai, H. Synthetic Routes to Mercury Chalcogenide Quantum Dots. J. Mater. Chem. C 2018, 6, 5097-5112.

(3) Nozik, A. J. Quantum Dot Solar Cells. Phys. E Low-Dimens. Syst. Nanostructures 2002, 14, 115-120.

(4) McDonald, S. A.; Konstantatos, G.; Zhang, S.; Cyr, P. W.; Klem, E. J. D.; Levina, L.; Sargent, E. H. Solution-Processed PbS Quantum Dot Infrared Photodetectors and Photovoltaics. Nat. Mater. 2005, 4, 138-142.

(5) Hafiz, S. B.; Scimeca, M.; Sahu, A.; Ko, D.-K. Colloidal Quantum Dots for Thermal Infrared Sensing and Imaging. Nano Converg. 2019, 6, 7.

(6) Livache, C.; Martinez, B.; Goubet, N.; Ramade, J.; Lhuillier, E. Road Map for Nanocrystal Based Infrared Photodetectors. Front. Chem. 2018, 6, 575.

(7) Lhuillier, E.; Guyot-Sionnest, P. Recent Progresses in Mid Infrared Nanocrystal Optoelectronics. IEEE J. Sel. Top. Quantum Electron. 2017, 23, 6000208.

(8) Böberl, M.; Kovalenko, M. V.; Gamerith, S.; List, E. J. W.; Heiss, W. Inkjet-Printed Nanocrystal Photodetectors Operating up to $3 \mu \mathrm{m}$ Wavelengths. Adv. Mater. 2007, 19, 3574-3578.

(9) Cryer, M. E.; Halpert, J. E. $300 \mathrm{~nm}$ Spectral Resolution in the Mid-Infrared with Robust, High Responsivity Flexible Colloidal Quantum Dot Devices at Room Temperature. ACS Photonics 2018, 5, 3009-3015. 
(10) Chu, A.; Martinez, B.; Ferré, S.; Noguier, V.; Gréboval, C.; Livache, C.; Qu, J.; Prado, Y.; Casaretto, N.; Goubet, N. et al HgTe Nanocrystals for SWIR Detection and Their Integration up to the Focal Plane Array. ACS Appl. Mater. Interfaces 2019, 11, 33116-33123.

(11) Chatterjee, A.; Pendyala, N. B.; Jagtap, A.; Rao, K. S. R. K. Uncooled Mid-Wave Infrared Focal Plane Array Using Band Gap Engineered Mercury Cadmium Telluride Quantum Dot Coated Silicon ROIC. E-J. Surf. Sci. Nanotechnol. 2019, 17, 95-100.

(12) Buurma, C.; Pimpinella, R. E.; Ciani, A. J.; Feldman, J. S.; Grein, C. H.; Guyot-Sionnest, P. MWIR Imaging with Low Cost Colloidal Quantum Dot Films. In Optical Sensing, Imaging, and Photon Counting: Nanostructured Devices and Applications 2016; International Society for Optics and Photonics, 2016; Vol. 9933, p 993303.

(13) Lhuillier, E.; Scarafagio, M.; Hease, P.; Nadal, B.; Aubin, H.; Xu, X. Z.; Lequeux, N.; Patriarche, G.; Ithurria, S.; Dubertret, B. Infrared Photodetection Based on Colloidal Quantum-Dot Films with High Mobility and Optical Absorption up to THz. Nano Lett. 2016, 16, 1282-1286.

(14) Goubet, N.; Jagtap, A.; Livache, C.; Martinez, B.; Portalès, H.; Xu, X. Z.; Lobo, R. P. S. M.; Dubertret, B.; Lhuillier, E. Terahertz HgTe Nanocrystals: Beyond Confinement. J. Am. Chem. Soc. 2018, 140, 5033-5036.

(15) Izquierdo, E.; Robin, A.; Keuleyan, S.; Lequeux, N.; Lhuillier, E.; Ithurria, S. Strongly Confined HgTe 2D Nanoplatelets as Narrow Near-Infrared Emitters. J. Am. Chem. Soc. 2016, 138, 10496-10501.

(16) Tenney, S. M.; Vilchez, V.; Sonnleitner, M. L.; Huang, C.; Friedman, H. C.; Shin, A. J.; Atallah, T. L.; Deshmukh, A. P.; Ithurria, S.; Caram, J. R. Mercury Chalcogenide Nanoplatelet-Quantum Dot Heterostructures as a New Class of Continuously Tunable Bright Shortwave Infrared Emitters. J. Phys. Chem. Lett. 2020, 11, 3473-3480.

(17) Chang, Y.-C.; Schulman, J. N.; Bastard, G.; Guldner, Y.; Voos, M. Effects of Quasi-Interface States in HgTe-CdTe Superlattices. Phys. Rev. B 1985, 31, 2557-2560.

(18) Rogalski, A. HgCdTe Infrared Detector Material: History, Status and Outlook. Rep. Prog. Phys. 2005, 68, 2267-2336.

(19) Orlowski, N.; Augustin, J.; Gołacki, Z.; Janowitz, C.; Manzke, R. Direct Evidence for the Inverted Band Structure of HgTe. Phys. Rev. B 2000, 61, R5058-R5061.

(20) Bernevig, B. A.; Hughes, T. L.; Zhang, S.-C. Quantum Spin Hall Effect and Topological Phase Transition in HgTe Quantum Wells. Science 2006, 314, 1757-1761.

(21) König, M.; Wiedmann, S.; Brüne, C.; Roth, A.; Buhmann, H.; Molenkamp, L. W.; Qi, X.-L.; Zhang, S.C. Quantum Spin Hall Insulator State in HgTe Quantum Wells. Science 2007, 318, 766-770.

(22) Leubner, P.; Lunczer, L.; Brüne, C.; Buhmann, H.; Molenkamp, L. W. Strain Engineering of the Band Gap of HgTe Quantum Wells Using Superlattice Virtual Substrates. Phys. Rev. Lett. 2016, 117, 086403.

(23) Lhuillier, E.; Keuleyan, S.; Guyot-Sionnest, P. Optical Properties of HgTe Colloidal Quantum Dots. Nanotechnology 2012, 23, 175705.

(24) Chen, M.; Guyot-Sionnest, P. Reversible Electrochemistry of Mercury Chalcogenide Colloidal Quantum Dot Films. ACS Nano 2017, 11, 4165-4173.

(25) Hudson, M. H.; Chen, M.; Kamysbayev, V.; Janke, E. M.; Lan, X.; Allan, G.; Delerue, C.; Lee, B.; Guyot-Sionnest, P.; Talapin, D. V. Conduction Band Fine Structure in Colloidal HgTe Quantum Dots. ACS Nano 2018, 12, 9397-9404.

(26) Allan, G.; Delerue, C. Tight-Binding Calculations of the Optical Properties of HgTe Nanocrystals. Phys. Rev. B 2012, 86, 165437.

(27) Keuleyan, S. E.; Guyot-Sionnest, P.; Delerue, C.; Allan, G. Mercury Telluride Colloidal Quantum Dots: Electronic Structure, Size-Dependent Spectra, and Photocurrent Detection up to $12 \mu \mathrm{m}$. ACS Nano 2014, 8, 8676-8682.

(28) Chen, M.; Guyot-Sionnest, P. Reversible Electrochemistry of Mercury Chalcogenide Colloidal Quantum Dot Films. ACS Nano 2017, 11, 4165-4173.

(29) Jagtap, A.; Martinez, B.; Goubet, N.; Chu, A.; Livache, C.; Gréboval, C.; Ramade, J.; Amelot, D.; Trousset, P.; Triboulin, A. et al Design of a Unipolar Barrier for a Nanocrystal-Based Short-Wave Infrared Photodiode. ACS Photonics 2018, 5, 4569-4576.

(30) Izquierdo, E.; Dufour, M.; Chu, A.; Livache, C.; Martinez, B.; Amelot, D.; Patriarche, G.; Lequeux, N.; Lhuillier, E.; Ithurria, S. Coupled HgSe Colloidal Quantum Wells through a Tunable Barrier: A Strategy To Uncouple Optical and Transport Band Gap. Chem. Mater. 2018, 30, 4065-4072.

(31) Gréboval, C.; Izquierdo, E.; Livache, C.; Martinez, B.; Dufour, M.; Goubet, N.; Moghaddam, N.; Qu, J.; Chu, A.; Ramade, J. et al Impact of Dimensionality and Confinement on the Electronic Properties of Mercury Chalcogenide Nanocrystals. Nanoscale 2019, 11, 3905-3915. 
(32) Goubet, N.; Livache, C.; Martinez, B.; Xu, X. Z.; Ithurria, S.; Royer, S.; Cruguel, H.; Patriarche, G.; Ouerghi, A.; Silly, M. et al Wave-Function Engineering in HgSe/HgTe Colloidal Heterostructures To Enhance Mid-Infrared Photoconductive Properties. Nano Lett. 2018, 18, 4590-4597.

(33) Pedetti, S.; Nadal, B.; Lhuillier, E.; Mahler, B.; Bouet, C.; Abécassis, B.; Xu, X.; Dubertret, B. Optimized Synthesis of CdTe Nanoplatelets and Photoresponse of CdTe Nanoplatelets Films. Chem. Mater. 2013, 25, 2455-2462.

(34) Pedetti, S.; Ithurria, S.; Heuclin, H.; Patriarche, G.; Dubertret, B. Type-II CdSe/CdTe Core/Crown Semiconductor Nanoplatelets. J. Am. Chem. Soc. 2014, 136, 16430-16438.

(35) Śniadower, L.; Psoda, M.; Gałązka, R. R. X-Ray Measurements of Lattice Dilatation in HgTe. Phys. Status Solidi B 1968, 28, K121-K123.

(36) Keuleyan, S.; Lhuillier, E.; Guyot-Sionnest, P. Synthesis of Colloidal HgTe Quantum Dots for Narrow Mid-IR Emission and Detection. J. Am. Chem. Soc. 2011, 133, 16422-16424.

(37) Geiregat, P.; Houtepen, A. J.; Sagar, L. K.; Infante, I.; Zapata, F.; Grigel, V.; Allan, G.; Delerue, C.; Van Thourhout, D.; Hens, Z. Continuous-Wave Infrared Optical Gain and Amplified Spontaneous Emission at Ultralow Threshold by Colloidal HgTe Quantum Dots. Nat. Mater. 2018, 17, 35-42.

(38) Guenzer, C. S.; Bienenstock, A. Temperature Dependence of the HgTe Band Gap. Phys. Rev. B 1973, 8, 4655-4667.

(39) Dobrowolska, M.; Mycielski, A.; Dobrowolski, W. Determination of Temperature Dependence of Energy Gap in HgTe by Oscillatory Magnetotransmission Measurements. Solid State Commun. 1978, 27, 1233-1235.

(40) Fonthal, G.; Tirado-Mejía, L.; Marín-Hurtado, J. I.; Ariza-Calderón, H.; Mendoza-Alvarez, J. G. Temperature Dependence of the Band Gap Energy of Crystalline CdTe. J. Phys. Chem. Solids 2000, 61, 579-583.

(41) Ithurria, S.; Tessier, M. D.; Mahler, B.; Lobo, R. P. S. M.; Dubertret, B.; Efros, A. L. Colloidal Nanoplatelets with Two-Dimensional Electronic Structure. Nat. Mater. 2011, 10, 936-941.

(42) Kushavah, D.; Mohapatra, P. K.; Ghosh, P. Reduced Carrier Trapping in CdSe/ZnS/CdSe Heterostructure Quantum Dots Inferred from Temperature Dependent Spectral Studies. Phys. E LowDimens. Syst. Nanostructures 2018, 102, 58-65.

(43) Ramade, J.; Andriambariarijaona, L. M.; Steinmetz, V.; Goubet, N.; Legrand, L.; Barisien, T.; Bernardot, F.; Testelin, C.; Lhuillier, E.; Bramati, A. et al Exciton-Phonon Coupling in a $\mathrm{CsPbBr}_{3} \mathrm{Single}$ Nanocrystal. Appl. Phys. Lett. 2018, 112, 072104.

(44) Olkhovets, A.; Hsu, R.-C.; Lipovskii, A.; Wise, F. W. Size-Dependent Temperature Variation of the Energy Gap in Lead-Salt Quantum Dots. Phys. Rev. Lett. 1998, 81, 3539-3542.

(45) Livache, C.; Goubet, N.; Gréboval, C.; Martinez, B.; Ramade, J.; Qu, J.; Triboulin, A.; Cruguel, H.; Baptiste, B.; Klotz, S. et al Effect of Pressure on Interband and Intraband Transition of Mercury Chalcogenide Quantum Dots. J. Phys. Chem. C 2019, 123, 13122-13130.

(46) Wright, N. G.; McMahon, M. I.; Nelmes, R. J.; San-Miguel, A. Crystal Structure of the Cinnabar Phase of HgTe. Phys. Rev. B 1993, 48, 13111-13114.

(47) San-Miguel, A.; Wright, N. G.; McMahon, M. I.; Nelmes, R. J. Pressure Evolution of the Cinnabar Phase of HgTe. Phys. Rev. B 1995, 51, 8731-8736.

(48) Düz, I.; Erdem, I.; Ozdemir Kart, S.; Kuzucu, V. First Principles Investigations of $\mathrm{HgX}(\mathrm{X}=\mathrm{S}$, Se and Te). Arch Mater Sci Eng 2016, 79, 5-11.

(49) Meulenberg, R. W.; Strouse, G. F. Pressure-Induced Electronic Coupling in CdSe Semiconductor Quantum Dots. Phys. Rev. B 2002, 66, 035317.

(50) Li, B.; Liu, W.; Zhu, X.; Lin, S.; Yang, Y.; Yang, Q.; Jin, P. Pressure-Dependent Photoluminescence of CdSe/ZnS Quantum Dots: Critical Point of Different Pressure Regimes. Phys. Lett. A 2019, 383, 14831486.

(51) Fan, H. M.; Ni, Z. H.; Feng, Y. P.; Fan, X. F.; Kuo, J. L.; Shen, Z. X.; Zou, B. S. High Pressure Photoluminescence and Raman Investigations of CdSe/ZnS Core/Shell Quantum Dots. Appl. Phys. Lett. 2007, 90, 021921.

(52) Svane, A.; Christensen, N. E.; Cardona, M.; Chantis, A. N.; van Schilfgaarde, M.; Kotani, T. Quasiparticle Band Structures of $\beta-\mathrm{HgS}, \mathrm{HgSe}$, and HgTe. Phys. Rev. B 2011, 84, 205205.

(53) Man, P.; Pan, D. S. Infrared Absorption in HgTe. Phys. Rev. B 1991, 44, 8745-8758.

(54) Pokatilov, E. P.; Fonoberov, V. A.; Fomin, V. M.; Devreese, J. T. Electron and Hole States in Quantum Dot Quantum Wells within a Spherical Eight-Band Model. Phys. Rev. B 2001, 64, 245329.

(55) El kurdi, M.; Fishman, G.; Sauvage, S.; Boucaud, P. Comparison between 6-Band and 14-Band k.p Formalisms in SiGe/Si Heterostructures. Phys. Rev. B 2003, 68, 165333.

(56) Laurenti, J. P.; Camassel, J.; Bouhemadou, A.; Toulouse, B.; Legros, R.; Lusson, A. Temperature Dependence of the Fundamental Absorption Edge of Mercury Cadmium Telluride. J. Appl. Phys. 1990, 67, 6454-6460. 
TOC graphic

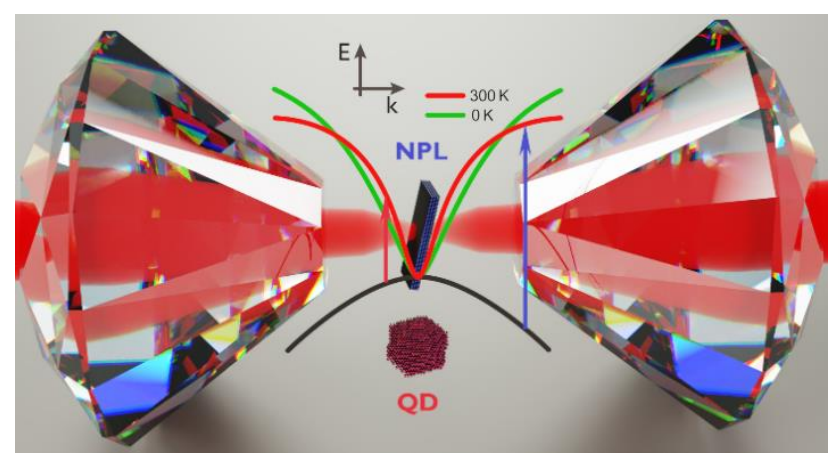

